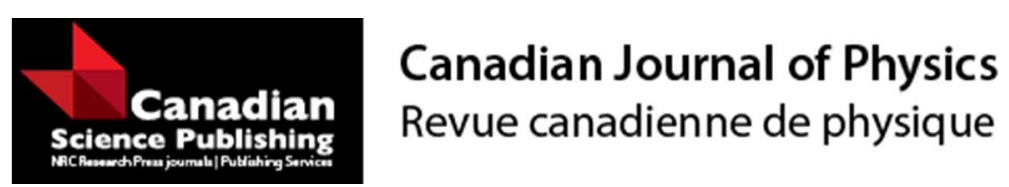

\title{
Reduction of the atomic wavefunction in the Stern-Gerlach magnetic field
}

\begin{tabular}{|r|l|}
\hline Journal: & Canadian Journal of Physics \\
\hline Manuscript ID: & cjp-2015-0031.R2 \\
\hline Manuscript Type: & Article \\
\hline Date Submitted by the Author: & 30 -Apr-2015 \\
\hline Complete List of Authors: & Devereux, Michael; Los Alamos National Laboratory (retired), \\
\hline Keyword: & $\begin{array}{l}\text { Stern-Gerlach experiment, Quantum measurement, Wavefunction } \\
\text { reduction, Discontinuous Schrodinger evolution, Stern-Gerlach } \\
\text { "interferometer" }\end{array}$ \\
\hline &
\end{tabular}

SCHOLARONE ${ }^{\mathrm{m}}$

Manuscripts 


\title{
Reduction of the atomic wavefunction in the Stern-Gerlach magnetic field \\ Michael Devereux ${ }^{a}$ \\ Los Alamos National Laboratory (Retired) \\ $137340^{\text {th }}$ St., Los Alamos, NM 87544
}

\begin{abstract}
Observation of two separated beam spots at a detection screen downstream of a SternGerlach magnet does not, in fact, demonstrate that the wavefunction of a neutral spin one-half particle has remained in a spin superposition while traveling through that magnetic field. The wavefunction may have been reduced to just one spin-direction eigenfunction, as David Bohm suggested, by immediate momentum and energy transfer with the magnet, rather than by subsequent, which-way determination at the screen. The same two beam spots at the detector screen will result. Einsteinian relativity, and the understanding of Schrödinger evolution applicability through a static potential, forbid continuation of a spin superposition through the Stern-Gerlach field. A calculation for single wavepacket development there conforms to observations from the Stern-Gerlach experiment. And several experiments corroborate immediate reduction to a single spin eigenfunction in the magnetic field. Additionally, Ramsey's separated, oscillating fields observations, and related experiments, do not rebut this understanding.
\end{abstract}

PACS 03.65.Ta 03.65.-w

\section{Introduction}

The familiar Stern-Gerlach (S.-G.) experiment [1,2] conceived more than ninety years ago, has remained a ubiquitous pedagogical paradigm and the canonical model for quantum measurement ever since. Though the S.-G. apparatus is unsophisticated by present standards, the observation of spin quantization which it enabled is of enduring value. Many recent physical theories exploring the beguiling mysteries of quantum measurement are founded on a detailed analysis of this experiment. And much research, positing continuous Schrödinger development of two separating wavepackets in the S.-G. magnetic field continues [3-6].

But we must reconsider that essential and widely-accepted tenet of existing Stern-Gerlach analysis. The wavefunction of a neutral, spinning particle traversing an S.-G. magnet is supposed to remain in a continuous superposition of spin directions until the particle's which-way position is detected at a downstream screen. But that prevailing ("orthodox") explanation, included in all our textbooks on the subject, certainly contradicts a foundational principle of Einsteinian relativity (special and general) - that energy transport must occur at finite speed. It also violates a basic understanding of when Schrödinger wavefunction evolution is applicable. Continuous Schrödinger wavefunction development would imply constant total energy of the quantum system considered, as it traveled through a static potential, like an S.-G. magnet. But, clearly, the total energy of a particle traversing the S.-G. apparatus is not constant.

Feynman and other prominent quantum theorists have told us that energy exchange with an apparatus is the process which distinguishes one among many superposed quantum paths, resulting in just one such path. Thus, following energy exchange with the S.-G. magnet, transferring field quanta, there must be just a single wavepacket representing the spinning particle. A computation for development of that single wavepacket within the magnetic field agrees with the observations of Stern and Gerlach. And,

a e-mail: $\underline{\text { d bar_x@enturylink.net }}$ 
no empirical evidence substantiates continuation of a spin superposition through the S.-G. magnet, though there are several experiments which deny that explanation.

In section 2 it's shown that our accepted explanation of continuous evolution of two, simultaneous, superposed atomic wavepackets through the S.-G. magnetic field is not credible, and is not supported by experimental evidence. Energy exchange within that field implies reduction to a single eigenfunction there. In section 3, historical development of the S.-G. theory is reviewed, and a realistic solution for the Schrödinger equation, and wavefunction evolution during field-quantum exchange, is presented. Section 4 depicts several simple experiments which substantiate reduction, via energy exchange, of the atomic spin superposition to a single path amplitude and spin eigenfunction within the magnet. A few, brief comments are offered in section 5. An appendix, finally, explains that the device called a Stern-Gerlach "interferometer", and related experiments, do not, in fact, measure interference between an atom's two purported, superposed wavepackets.

\section{Energy transfer and quantum measurement}

Observation shows that each individual physical particle that strikes the S.-G. detection screen has acquired an additional, transverse kinetic energy (without corresponding loss of its incident, longitudinal kinetic energy), pushing it to either one side, or the other, of the particle's initial direction entering the magnet. Each of the two separated traces on the detection screen results from a distinctive momentum transfer to the particle from the magnetic field. Obviously, that momentum, and kinetic energy, was gained in the S.-G. field, not in the vacuum between field and screen. According to Einstein, there can be no instantaneous transfer of that additional, macroscopic energy, from the magnet to the spinning particle (no action-at-a-distance), at the moment of particle detection at the screen. Nor can the particle's wavefunction deliver that physical energy from the magnetic field to the detector. ${ }^{1}$

Wavefunctions, of course, have no mass, $\mathrm{m}=\mathrm{E} / \mathrm{c}^{2}$, nor energy, E. Instead, macroscopic energy, in the form of innumerable field quanta, is transferred to the particle, repeatedly kicking it just one way as it travels within the magnetic field. That additional, discrete, kinetic energy must be carried at a finite speed, from the magnet to the detector by each atom, just as the initial longitudinal energy is done. In the mathematical formalism of quantum theory, such a situation is described as a statistical mixture of atomic energy (and spin) states, not a quantum superposition. The likely result of a quantum energy (or spin) measurement is completely determined at that point. Moreover, the mixture represents an immediate disentanglement of internal spin from external wavepacket dependence.

Schrödinger's equation takes on an especially tractable form if the potential term has no time dependence, as is the case for a static Stern-Gerlach magnet. Schrödinger's equation becomes separable, with terms dependent only on time equal to terms only dependent on position. (Section 3.) So, each side of the equation must equal a constant, which, in the case of a neutral, spinning atom, traversing an S.-G. magnet, would be the conserved total energy of the atom. Because that atom's total energy is not, in fact, conserved, continuous Schrödinger evolution cannot describe the atom's wavefunction development throughout the magnetic field. Instead, a new interval of Schrödinger time development is initiated at each magnetic-field, quantum transfer, which specifies the new initial conditions.

Richard Feynman said that the quantum wavefunction of some physical object, such as a neutral, spinning particle in the S.-G. apparatus, represents the sum of probability amplitudes for each indistinguishable path that object might take [7]. But he showed that energy transfer between the object on one of those paths, and an apparatus, makes that particular path distinguishable. Feynman cites the example of a photon scattered from an electron, just as the electron passes through one of the two slits in a screen [8]. Energy exchange with the scattered photon reduces the electron wavefunction to one path amplitude, thus destroying the superposition, resulting in a statistical mixture. A polarized neutron experiment, described in section 4, is particularly persuasive in this regard. Similarly, an energetic quantum transferred from the S.-G. magnetic field to the spinning particle, kicks the magnet (apparatus)

\footnotetext{
${ }^{1}$ We note, with care, that prohibition against instantaneous energy transmission does not exclude the sort of influence which appears to determine the non-local correlation of spin directions in an EPR-Aspect experiment.
} 
in the direction opposite to that imparted to the particle, measuring which path is taken, and destroying the initial path superposition. David Bohm, too, in his estimable book on quantum mechanics, envisioned the Stern-Gerlach spin measurement as a momentum exchange. "Thus, it would be possible in principle to measure the spin by measuring the momentum transmitted to the particle by the magnetic field," he said [9].

Clearly, there exist situations in which a quantum wavepacket is continuously divided into parts which can be recombined to display interference. Passage of a single photon through two narrow slits is one such. Bragg scattering of electrons, or neutrons, from a crystal [10], is another instance. Likewise, the wavefunction of an atom, or macromolecule, may be divided by a micro-fabricated grating, or an optical grating, then recombined in an interference pattern [11]. Because there is no energy transfer to another physical object that distinguishes which path is taken, the wavefunction is not reduced in those cases, and interference may be observed. Within the S.-G. magnetic field, however, there is momentum and energy exchange, and the path of the spinning particle is clearly distinguished.

Moreover, from the beginnings of the formulation of quantum theory, we've known that Schrödinger evolution of the wavefunction is valid only for closed, constant-energy systems [12]. Wigner taught that "In quantum mechanics, as in classical physics, we postulate the existence of isolated systems. In both theories, if a complete description of an isolated system is given at one time, a complete description for any other time is uniquely determined as long as the system remains isolated - i.e., is not influenced by any other system" [13]. And, Zurek made this same principle central to development of his decoherence theory [14]. When an energetic quantum is absorbed, an interval of continuous Schrödinger evolution ceases, and a new interval begins, from new initial conditions supplied by that quantum transfer; as within the S.-G. magnet.

Significantly, there is no experimental evidence to confirm continuous wavefunction development throughout the magnetic field, though textbooks, and physics researchers, all seem to agree on it. The same two beam spots would appear on the detection screen whether the wavefunction remains in a spin superposition through the field, or is, instead, reduced to a single spin eigenfunction by the first field quantum it absorbs. There are, however, experiments, described below, which do show that quantum energy absorption is the mechanism for immediately distinguishing which path the spinning particle travels through the S.-G. field, so reducing the energy superposition to a single eigenfuction.

\section{Stern-Gerlach analysis, historically}

As taught by our textbooks, and confirmed by innumerable observations, the S.-G. apparatus will split a stream of neutral, spin one-half, silver atoms in two, deflecting half of them to one side, and half to the other side of their original path, entering the magnet. Suppose a silver atom is traveling in the positive y direction entering a Stern-Gerlach magnet, depicted in Fig. 1. The incident stream of atoms is confined to a small cross section centered on the y axis. Because the actual magnet is very long compared to the gap between pole pieces, we assume that within the magnet the field, $\mathbf{B}$, has no y dependence and that $B_{y} \equiv 0$. Very close to the $\mathrm{y}-\mathrm{z}$ plane the non-uniform field will be predominantly in the $\mathrm{z}$ direction. As has often been done in the past $[15,16]$ one might then posit a simple field, $\mathbf{B}_{\mathbf{S}}=\left(0,0, B_{z}(x, z)\right)$, encountered by the atoms.

A silver atom is a spin one-half particle because of its single valence electron. Classically, the force on each of those atoms is $\mathbf{F}=-\nabla\left(\boldsymbol{\mu}_{A} \bullet \mathbf{B}\right)$, where $\boldsymbol{\mu}_{\square}$ is the atom's magnetic moment, which has a $\mathrm{z}$ component, $\mu_{z}=-\frac{e}{m_{e}} s_{z}$, and a spin component of $s_{z}=\frac{\hbar}{2}$, where $\mathrm{e}$ is the electron's charge and $\mathrm{m}_{\mathrm{e}}$ is its mass. Thus, for the simple field $\mathbf{B}_{\mathrm{s}}$, if $\partial_{x} B_{z}\left\langle\left\langle\partial_{z} B_{z}\right.\right.$, the force would be $\pm \mu_{z} \partial_{z} B_{z}$, which would account for half the atoms being directed toward $+\mathrm{z}$ and half the other way, just as recorded by an S.-G. observation. Figure 2 depicts the pattern of silver atoms detected by Stern and Gerlach in 1922 [2]. Deflection occurs only along the $\mathrm{z}$ axis.In more recent times some researchers have attempted to give a completely quantum-mechanical description of the Stern-Gerlach experiment. But, in the ninety years since the original S.-G. observations, no one seems to have considered the possibility that immediate energy 
exchange within the magnetic field must therefore result in abrupt reduction of the atom's magnetic moment to either $-\frac{e \hbar}{2 m_{e}} \hat{k}$ or $+\frac{e \hbar}{2 m_{e}} \hat{k}$. It would seem obvious that quantum measurement is the exchange of information between an examined physical object and an apparatus. Any energy transfer between the two will carry information about the shift in energy state of each object, identifying energy exchange as a quantum measurement. ${ }^{2}$

Here, we will consistently interpret the quantum wavefunction as Born taught: thus, $\Psi^{*}(q) \Psi(q) d q$ is the probability that the physical system represented by $\Psi(q)$ will be found to have a value between $\mathrm{q}$ and $\mathrm{q}+\mathrm{dq}$ if that property is measured [18]. For the quantum-mechanical description of the S.-G. experiment one may write the spin portion of the atom's measured state vector, before that atom encounters the S.-G. magnetic field, as $|\chi\rangle=\frac{1}{\sqrt{2}}\left(\left|\uparrow_{z}\right\rangle+\left|\downarrow_{z}\right\rangle\right)$, anticipating the measurement result ${ }^{3}$.

David Bohm described the spinning atom traversing the S.-G. magnet as a wavepacket, $\psi(\mathbf{r}, t)$, influenced by the simplistic field $\mathbf{B}_{\mathbf{S}}$ [19]. (He did recognize that $\mathbf{B}_{\mathbf{S}}$ does not satisfy Maxwell's equation, $\nabla \bullet \mathbf{B}=0$, but suggested that wavepacket motion in the $\mathrm{x}$ direction is not of interest in this situation.) The interaction energy between particle and field, then, would be $\mathrm{V}=\boldsymbol{\mu}_{\square} \bullet \mathbf{B}_{s}$, which can be expanded about $\mathrm{z}$ $=0, V \approx \mu_{z} B_{z(z=0)}+z \mu_{z}\left(\frac{\partial B_{z}}{\partial z}\right)_{(z=0)}$. Unfortunately, like other researchers, he presumed a continued separation of the wavepacket in two pieces within the field, yielding a purported, "entangled" state, $\Psi(\mathbf{r}, t, s)=\frac{1}{\sqrt{2}}\left[\psi_{\uparrow}(\mathbf{r}, t)\left|\uparrow_{z}\right\rangle+\psi_{\downarrow}(\mathbf{r}, t)\left|\downarrow_{z}\right\rangle\right]$. Then, those pieces would continuously develop via Schrödinger evolution through the apparatus. The Schrödinger equation is tractable with these simplifying assumptions, and portrays the two wavepackets as separating in opposite directions along the $\mathrm{z}$ axis.

Scully, Shea, and McCullen (SSM) used the Stern-Gerlach experiment to examine quantum measurement [20]. They portrayed the particle wavefunction, traveling through the magnetic field, as a minimum uncertainty wavepacket, with spin, and supposed, as is typically done, that it is immediately split into two, superposed parts in the field. For time development of those two wavepackets they employed the Kennard propagator technique [21]. And they chose $\mathbf{B}_{\mathbf{S}}$ for their magnetic field, which does not, therefore, satisfy Maxwell's equations.

SSM proposed installing a light, bi-level atom along one possible path through the S.-G. magnet as measuring apparatus. If the spinning particle travels that way, it will excite the atom; otherwise not. SSM said that if the spinning particle is much heavier than the atom, there would be negligible momentum transfer from the measured system to the apparatus, and the wavefunction of that spinning particle is said to be continuous (unperturbed) in the interaction. SSM could then show that the density matrix of the system-apparatus would be nearly diagonalized.

It must be clear, though, that however much the mass disparity between system and apparatus, there is always the same quanitized energy, equal to the difference between the atom's ground and excited level, transferred from the spinning particle to the atom at collision. And, every unique energy eigenvalue of the particle is associated with a different, linearly-independent eigenfunction, which specifies a different measured particle state. So, one is justified in denying the continuous development of the system wavefunction at detection, essential to the SSM analysis. (I have expanded on this argument to criticize quantum eraser theory in general [22].)

\footnotetext{
${ }^{2}$ Elsewhere, I've critically examined the Szilard engine [17], which demonstrates the relationship between information and physical entropy. And, there I made the case in more detail that energy exchange must be a quantum measurement.

${ }^{3}$ It is equally important to recognize what the Born definition does not include. The wavefunction is not said to represent the likely present state of the physical system.
} 
Scully, Lamb, and Barut [23] extended this S.-G. analysis to a somewhat more authentic magnetic field, $\mathbf{B}=(-\mathrm{bx}, 0, \mathrm{bz})$, with $\mathrm{b}$ constant, though they posit a magnet which is very short in the beam direction, even compared to the gap between pole pieces. And, no propagated solutions are calculated.

Typically, a real S.-G. magnet has a gap that is much smaller than the magnet's extension along the beam direction, and is also symmetric about the $y-z$ plane, as in Fig. 1. So, $B_{z}$ is an even function of $x$ [24]. Then, near $\mathbf{x}=0$, where atoms enter the magnet, $\mathbf{F}=-\nabla(\mu \bullet \mathbf{B})=-\left(\mu_{x} \partial_{x} B_{x}, 0, \mu_{x} \partial_{z} B_{x}+\mu_{z} \partial_{z} B_{z}\right)$. Because $\nabla \bullet \mathbf{B}=0$, we get, $\mathbf{F}=\left(\mu_{x} \partial_{z} B_{z}, 0,-\mu_{x} \partial_{z} B_{x}-\mu_{z} \partial_{z} B_{z}\right)$. Since $\nabla \times \mathbf{B}=0$ also, $\partial_{z} B_{x}=\partial_{x} B_{z}=0$, and,

$$
\mathbf{F}=\left(\mu_{x} \partial_{z} B_{z}, 0,-\mu_{z} \partial_{z} B_{z}\right)
$$

In the classical picture one expects an incident atom's spin to be oriented randomly in the $\mathrm{x}-\mathrm{Z}$ plane, so this force would result in atoms being deflected isotropically in that plane. Observation, however, refutes that conclusion; deflection only occurs along the $\mathrm{z}$ axis. It can be shown that a consideration of the classical motion of precession would account for that discrepancy.

Atoms entering the S.-G. magnet are narrowly collimated along the y axis where they do experience a magnetic field that is very nearly parallel to $\hat{z}$. If the magnetic moments of those atoms remained randomly distributed upon entering the field (though quantum mechanics requires that this not be the case) precession of the atomic moment about $\mathbf{B}$ would occur. Classically, torque on the atom would be $\tau=\mu \times \mathbf{B}$ with $\dot{\mu}=\omega \times \mu$, and the atom would precess with the Larmour frequency $\omega=\frac{e}{2 m_{e}} \mathbf{B}$. To a very good approximation, near $\mathrm{x}=0, \omega_{x}=\omega_{y}=0$, so that $\boldsymbol{\omega} \square=\omega_{z} \hat{k}=\frac{e}{2 m_{e}} B_{z} \hat{k}$. Then,

$$
\dot{\mu}=\left(-\omega \mu_{y}, \omega \mu_{x}, 0\right)
$$

yielding the solution [24],

$$
\begin{aligned}
& \mu_{x}(t)=\mu_{x}(0) \cos \omega t-\mu_{y}(0) \sin \omega t, \\
& \mu_{y}(t)=\mu_{x}(0) \sin \omega t+\mu_{y}(0) \cos \omega t, \text { and } \\
& \mu_{z}(t)=\mu_{z}(0) .
\end{aligned}
$$

From Eq. (1), the force experienced by an atom is,

$$
\begin{aligned}
& F_{x}(t)=\left(\mu_{x}(0) \cos \omega t-\mu_{y}(0) \sin \omega t\right) \partial_{z} B_{z}, \\
& F_{y}(t)=0, \text { and } \\
& F_{z}(t)=-\mu_{z}(0) \partial_{z} B_{z} .
\end{aligned}
$$

For a silver atom in a field of about $4 \times 10^{3}$ Gauss, $\omega \approx 10^{11} \mathrm{rad} . / \mathrm{sec}$. An atom, traveling at about $1000 \mathrm{~m} / \mathrm{sec}$. would require about $10^{-4} \mathrm{sec}$. to pass through a magnet $10 \mathrm{~cm}$. long. Thus, from classical physics, due to precession, the $\mathrm{x}$-direction force on the atom would average zero. This could account for the observed deflection of atoms, exclusively toward either $+\hat{z}$ or $-\hat{z}$.

Later, Platt showed how to incorporate these classical ideas into a quantum mechanical description of the Stern-Gerlach experiment. He employed a field which is realistic, but, unfortunately, he also assumed, as, it seems, is always done, a continuing superposition of the two spin eigenfunctions [25]. He represented the magnetic field by the more credible formula, $\mathbf{B}_{R}=\left(-b x, 0, b z+B_{0}\right)$, with $\mathrm{B}_{0}$ and $\mathrm{b}$ constant, which does satisfy Maxwell's equations. A more realistic model of the field, such as this, has now become usual in S-G analyses, permitting an increasingly perspicacious comparison with S-G observations. In fact, some researchers, familiar with neutron experiments, have suggested that modeling 
the fringing fields at the magnet's entrance and exit is important for calculating changes in the particle's potential energy, $\boldsymbol{\mu} \bullet \mathbf{B}$. However, for analysis of the Stern-Gerlach experiment, because $\mathbf{B}=(0,0,0)$ before, and just after those fringing fields, there can be no change in that potential crossing the entire magnet. Zero minus zero yields zero. That's all one needs to know.

The Schrödinger equation, $i \hbar \frac{\partial}{\partial t}|\Psi\rangle=\mathbf{H}|\Psi\rangle$, has a Hamiltonian operator, $\mathbf{H}=\frac{\mathbf{P}^{2}}{2 m_{A}}-\frac{e}{m_{e}} \sigma \bullet \mathbf{B}$, where $\mathrm{m}_{\mathrm{A}}$ is the atom's mass, and $\boldsymbol{\sigma}$ is the vector spin operator, implying, of course, a spinor coupling between $\mathbf{B}$ and the atom's magnetic moment, $\mu$. For spin one-half particles, $\sigma=\frac{\hbar}{2}\left(\begin{array}{ll}0 & 1 \\ 1 & 0\end{array}\right) \hat{i}+\frac{\hbar}{2}\left(\begin{array}{cc}0 & -i \\ i & 0\end{array}\right) \hat{j}+\frac{\hbar}{2}\left(\begin{array}{cc}1 & 0 \\ 0 & -1\end{array}\right) \hat{k}$. If it were true that the atom's wavefunction remained in a spin superposition throughout this magnetic field, $\Psi(\mathbf{r}, t, s)=\left(\begin{array}{l}\Psi_{\uparrow}(\mathbf{r}, t, s) \\ \Psi_{\downarrow}(\mathbf{r}, t, s)\end{array}\right)$, with $\Psi_{\uparrow}(\mathbf{r}, t, s)=\psi_{\uparrow}(\mathbf{r}, t)|\uparrow\rangle$, etc, one would write the coupled spinor equations for Schrödinger time development as,

$$
\begin{aligned}
& i \hbar \frac{\partial \psi_{\uparrow}}{\partial t}=-\frac{\hbar^{2}}{2 m_{A}} \nabla^{2} \psi_{\uparrow}+\frac{e}{m_{e}} \frac{\hbar}{2} b x \psi_{\downarrow}-\frac{e}{m_{e}} \frac{\hbar}{2}\left(b z+B_{0}\right) \psi_{\uparrow}, \text { and } \\
& i \hbar \frac{\partial \psi_{\downarrow}}{\partial t}=-\frac{\hbar^{2}}{2 m_{A}} \nabla^{2} \psi_{\downarrow}+\frac{e}{m_{e}} \frac{\hbar}{2} b x \psi_{\uparrow}+\frac{e}{m_{e}} \frac{\hbar}{2}\left(b z+B_{0}\right) \psi_{\downarrow} .
\end{aligned}
$$

We see that the two equations are coupled through the $\mathrm{x}$ component of the non-uniform field. And, classically, there would be precession of a magnetic moment about the $\mathbf{B}$ field at the Larmour frequency, $\omega_{L}=\frac{e}{2 m_{e}} B$. But quantum mechanics insists that the atom's magnetic moment can exist in only two directions: parallel or anti-parallel to B. So, there is actually no physical oscillation of an atom's magnetic moment about B. But there is, nevertheless, an oscillatory component in the atom's wavefunction at the Larmour frequency, predicting the probability of energy exchange with an oscillatory magnetic field. (See Appendix.) To distinguish motion of the wavepacket that is persistent relative to those faster oscillations, one may write the wavepackets as $\psi_{\uparrow}(\mathbf{r}, t)=\bar{\psi}_{\uparrow}(\mathbf{r}, t) e^{i \omega_{L} t}$ and $\psi_{\downarrow}(\mathbf{r}, t)=\bar{\psi}_{\downarrow}(\mathbf{r}, t) e^{-i \omega_{L} t}$. Then, Schrödinger's equation, after dividing by the appropriate exponential, becomes,

$$
\begin{aligned}
& i \hbar \frac{\partial \bar{\psi}_{\uparrow}}{\partial t}-\hbar \omega_{L} \bar{\psi}_{\uparrow}=-\frac{\hbar^{2}}{2 m_{A}} \nabla^{2} \bar{\psi}_{\uparrow}+\frac{e}{m_{e}} \frac{\hbar}{2} b x \bar{\psi}_{\downarrow} e^{-2 i \omega_{L} t}-\frac{e}{m_{e}} \frac{\hbar}{2}\left(b z+B_{0}\right) \bar{\psi}_{\uparrow}, \text { and } \\
& i \hbar \frac{\partial \bar{\psi}_{\downarrow}}{\partial t}+\hbar \omega_{L} \bar{\psi}_{\downarrow}=-\frac{\hbar^{2}}{2 m_{A}} \nabla^{2} \bar{\psi}_{\downarrow}+\frac{e}{m_{e}} \frac{\hbar}{2} b x \bar{\psi}_{\uparrow} e^{2 i \omega_{L} t}+\frac{e}{m_{e}} \frac{\hbar}{2}\left(b z+B_{0}\right) \bar{\psi}_{\downarrow} .
\end{aligned}
$$

The coupling terms, linear in $\mathrm{x}$, oscillate rapidly relative to those other terms in the equations, and may be neglected. Because the Larmour frequency is $\omega_{L} \approx \frac{e}{2 m_{e}} B_{0}$, Schrödinger's equation is then,

$$
\begin{aligned}
& i \hbar \frac{\partial \bar{\psi}_{\uparrow}}{\partial t}=-\frac{\hbar^{2}}{2 m_{A}} \nabla^{2} \bar{\psi}_{\uparrow}-\mu_{B} b z \bar{\psi}_{\uparrow}, \text { and } \\
& i \hbar \frac{\partial \bar{\psi}_{\downarrow}}{\partial t}=-\frac{\hbar^{2}}{2 m_{A}} \nabla^{2} \bar{\psi}_{\downarrow}+\mu_{B} b z \bar{\psi}_{\downarrow},
\end{aligned}
$$


where $\mu_{B}=\frac{e \hbar}{2 m_{e}}$ is the Bohr magneton. Thus, these equations do describe separating wavepackets with a velocity component in opposite directions along z, predicting the observations of the S.-G. experiment.

Later, Home et al. [26] focused on the non-ideal nature of a quantum measurement as exemplified by the Stern-Gerlach experiment. Like other researchers, they surmise the continued existence of a spindirection superposititon throughout the magnetic field, and up to the detection screen. A solution to Schrödinger's equation for this divided wavepacket is enabled by decoupling the two spinor equations, based on "precession" and the use of coherent, internal states [27, 28].

Finally, it is also possible to employ numerical computations to model passage of a neutral, spin one-half particle through the S.-G. apparatus. Hsu et al., found that if the magnetic field is specified by the realistic formula, $\mathbf{B}_{\mathbf{R}}$, deriving a propagator is challenging [5]. Instead, they iteratively apply the timeevolution operator, $\mathbf{T}(t)=\exp \left(-\frac{i}{\hbar} \mathbf{H} t\right)$, using $\mathbf{B}_{\mathrm{R}}$ in the Hamiltonian, $\mathbf{H}$. That computation results in two spatial wavepackets beyond the magnet, $\left.\psi_{\uparrow}(\mathbf{r}, t) \uparrow_{z}\right\rangle$, and $\left.\psi_{\downarrow}(\mathbf{r}, t) \downarrow_{z}\right\rangle$, separating along the $\mathrm{z}$ direction, again predicting the observation of silver atoms from the historic S.-G. experiment.

Note, however, that such a computation does not demonstrate that the wavefunction of an actual silver atom will split into a superposition of two simultaneous, spin-direction wavepackets in the magnetic field. This computation, instead, is based on the ubiquitous assumption that the atom's wavefunction is not reduced (by momentum transfer) to a single wavepacket, with a single spin eigenfunction. Instead, such computations assume no wavefunction reduction, and so, then, calculate the time evolution for both $\left.\psi_{\uparrow}(\mathbf{r}, t) \uparrow_{z}\right\rangle$ and $\left.\psi_{\downarrow}(\mathbf{r}, t) \downarrow_{z}\right\rangle$ through the field, as if both wavepackets actually existed simultaneously.

It would appear that all researchers, since the time of Stern and Gerlach, have taken it for granted that a spin-direction superposititon, which predicts a spin measurement, persists throughout the magnetic field, and to the detector. But two separate traces on the detection screen indicate acquisition by each atom, and only within the magnetic field, of a well-defined, transverse kinetic energy. That energy must be transported at finite speed, by each atom, from the field to the detector. In the formalism of quantum theory a transverse energy (spin) measurement is then entirely determined, and one expresses that reality as a mixture of spin eigenfunctions, not a superposition.

If the wavefunction of the atom traveling throughout an S.-G. magnet were actually described by continuous Schrödinger evolution, the atom's total energy would be constant. Because Schrödinger's equation there is $i \hbar \frac{\partial \Psi(\mathbf{r}, t)}{\partial t}=-\frac{\hbar^{2}}{2 m_{A}} \nabla^{2} \Psi(\mathbf{r}, t)+V(\mathbf{r}) \Psi(\mathbf{r}, t)$, with the potential, $V(\mathbf{r})=-\frac{e}{m_{e}} \sigma \bullet \mathbf{B}(\mathbf{r})$, independent of time, there is a product wavefunction solution. Choose, $\Psi(\mathbf{r}, t)=\Phi(\mathbf{r}) \Theta(t)$. Then, $\frac{i \hbar}{\Theta(t)} \frac{\partial \Theta(t)}{\partial t}=\frac{1}{\Phi(\mathbf{r})}\left[-\frac{\hbar^{2}}{2 m_{A}} \nabla^{2} \Phi(\mathbf{r})+V(\mathbf{r}) \Phi(\mathbf{r})\right]$. The left side of the equation depends only on time, the right side only on position, so both sides must equal a constant, whose dimensions are energy, E. This gives a solution for the atomic wavefunction, $\Psi(\mathbf{r}, t)=e^{-\frac{i E t}{\hbar}} \Phi(\mathbf{r})$, and the total energy of the atom, E, would remain constant during continuous Schrödinger evolution throughout the field. But, because it's not constant, Schrödinger's equation can only describe wavefunction evolution during an interval between absorption of each magnetic quantum, with initial conditions dictated by that energy transfer.

This does make the actual quantum formulation between field-quantum transfers strikingly straightforward. The atomic wavefunction must be either $\left(\begin{array}{c}\Psi_{\uparrow}(\mathbf{r}, t, s) \\ 0\end{array}\right)$ or $\left(\begin{array}{c}0 \\ \Psi_{\downarrow}(\mathbf{r}, t, s)\end{array}\right)$, and the equation for Schrödinger evolution, for an isolated atom, is then either,

$$
i \hbar \frac{\partial \psi_{\uparrow}}{\partial t}=-\frac{\hbar^{2}}{2 m_{A}} \nabla^{2} \psi_{\uparrow}-\frac{e}{m_{e}} \frac{\hbar}{2}\left(b z+B_{0}\right) \psi_{\uparrow}, \text { or }
$$




$$
i \hbar \frac{\partial \psi_{\downarrow}}{\partial t}=-\frac{\hbar^{2}}{2 m_{A}} \nabla^{2} \psi_{\downarrow}+\frac{e}{m_{e}} \frac{\hbar}{2}\left(b z+B_{0}\right) \psi_{\downarrow}
$$

Again, there is a component of the atom's wavefunction that oscillates at the Larmour frequency, $\omega_{\mathrm{L}}$, predicting the probability of spin flip in an oscillating magnetic field. So, we take $\psi_{\uparrow}(\mathbf{r}, t)=\bar{\psi}_{\uparrow}(\mathbf{r}, t) e^{i \omega_{L} t}$ and $\psi_{\downarrow}(\mathbf{r}, t)=\bar{\psi}_{\downarrow}(\mathbf{r}, t) e^{-i \omega_{L} t}$ in order to illustrate linear motion of the wavepacket disassociated from these fast oscillations. Then, since $\omega_{L} \approx \frac{\mu_{A}}{\hbar} B_{0}$ and $\mu_{B}=\frac{e \hbar}{2 m_{e}}$, either

$$
\begin{aligned}
& i \hbar \frac{\partial \bar{\psi}_{\uparrow}}{\partial t}=-\frac{\hbar^{2}}{2 m_{A}} \nabla^{2} \bar{\psi}_{\uparrow}-\mu_{B} b z \bar{\psi}_{\uparrow}, \text { or } \\
& i \hbar \frac{\partial \bar{\psi}_{\downarrow}}{\partial t}=-\frac{\hbar^{2}}{2 m_{A}} \nabla^{2} \bar{\psi}_{\downarrow}+\mu_{B} b z \bar{\psi}_{\downarrow},
\end{aligned}
$$

describing a wavepacket with a transverse velocity toward either $+\hat{z}$ or $-\hat{z}$, as expected. Notice that, with a single wavepacket, there is no "entanglement" of internal spin components with wavepackets dependent on external freedom degrees.

The atom will experience innumerable energetic quantum exchanges traversing the field, and Eq. (9) specifies its single wavepacket development in between them. A convergent calculation for wavepacket development through the entire magnetic field is facilitated by recognition that the positionrepresentation wavepacket remains unchanged at each field quantum transfer. Between those quantum exchanges the wavepacket will evolve from time $\mathrm{t}_{0}$, to an infinitesimally later time, $\mathrm{t}_{0}+\square \mathrm{t}$, as,

$$
\bar{\psi}\left(\mathbf{r}, t_{0}+\delta t\right)=\left[\mathbf{1}-\frac{i}{\hbar} \delta \mathbf{t} \mathbf{H}\right] \bar{\psi}\left(\mathbf{r}, t_{0}\right) .
$$

We employ the realistic magnetic field, $\mathbf{B}_{\mathrm{R}}=\left(-\mathrm{bx}, 0, \mathrm{~B}_{0}+\mathrm{bz}\right)$ in the Hamiltonian, $\mathbf{H}$. Then, after a time, $\tau$, necessary to pass through the entire field, the evolved wavepacket is described by,

$$
\bar{\psi}\left(\mathbf{r}, t_{0}+\tau\right)=\lim _{n \rightarrow \infty}\left[\mathbf{1}-\frac{i}{\hbar} \frac{\tau}{n} \mathbf{H}\right]^{n} \bar{\psi}\left(\mathbf{r}, t_{0}\right)=\exp \left[\frac{-i}{\hbar} \tau \mathbf{H}\right] \bar{\psi}\left(\mathbf{r}, t_{0}\right) .
$$

This computation was performed by Hsu et al. (albeit, with the assumption of two simultaneous wavepackets), resulting in the transverse wavepacket motion toward either $+\hat{z}$ or $-\hat{z}$, conforming to S.G. observations, shown in their Fig. 7 [29].

In addition to the powerful, transverse field gradient created by an S-G magnet, there will be small, fringing fields at the entrance and exit, which also contain field gradients. But, no matter the form of the field anywhere in the magnet, the potential energy of a silver atom, $-\frac{e}{m_{e}} \boldsymbol{\sigma} \cdot \mathbf{B}$, is unchanged by passage through the field. It is zero well before the magnet, and zero beyond it.

The effect of the fringing fields on a neutral, spin one-half particle (a neutron in this case) was measured by Alefeld et al. [30]. They found that the kinetic energy of the neutron either increased by $\mu \mathrm{B}$, or decreased by $-\mu \mathrm{B}$, at the entrance of a uniform magnetic field, exactly compensating the shift in 
potential energy there. The opposite change in K.E. occurred at the exit, so conserving the neutron's total energy through a uniform field. ${ }^{4}$

Some physicists have made the argument [31] that Ramsey's separated, oscillating-fields device [32], and related atomic "interferometers" [33-36], refute the claim that energy exchange in the S.-G. magnetic field must result in a mixture of spin eigenfunctions, not a superposition. Incidentally, the researchers who have made observations with those devices have not recognized any immediate energy transfer to a spinning atom from an electromagnetic pulse. On the contrary, they interpret a $\pi / 2$ electromagnetic pulse as a means to create a quantum superposition of energy states, absent any immediate quantum energy transfer. It is an actual energy exchange which excludes the possibility of a continuing superposition.

The situation is subtle and interesting. (See the Appendix.) Ramsey's device, and some other atomic "interferometers", provide a sinusoidal data curve, similar to interference fringes, for the probability of an excited atomic state, versus, typically, variation in the frequency of the driving electromagnetic pulse. The sinusoidal curve of data collected with this device actually results from variation in the phase of the driving field versus the phase of the atom's oscillating, wavefunction component. Not from variation in the extent of overlap of two wavepackets, previously separated from a single atomic wavefunction. The extraordinary atomic fountain implemented by Kasevitch and Chu, [33] and utilized by Kasevich and his colleagues, [36] is based on this same principle. (Appendix.)

\section{Experiments}

A physical phenomenon with no empirical evidence to substantiate the explanation found in all our textbooks would seem to demand experimental test. And one can find experiments which support the understanding that energy exchanged between an observed atomic system and an energetic field is a quantum measurement which results in a statistical mixture of the energetic particle states, not a quantum superposition. Feynman [37], and later, Jauch [38], described a simple, limpid, particularly persuasive experiment, demonstrating this fact. It seems to have received much too little attention.

Consider a stream of polarized neutrons incident on a crystal with polarized nuclei. If a neutron exchanges spin, and energy, via collision, with a particular nucleus, that neutron's path is distinguished, and that scattering is incoherent. Observation shows that the neutron does not contribute to an interference pattern downstream. On the other hand, if no spin-flip occurs at collision, those neutrons can be seen to retain their original polarization, and the scattering is elastic. Only such elastically-scattered neutrons produce interference. Similarly, since there is energy exchanged between the S.-G. magnet and the atom which distinguishes the atom's path, we must expect a mixture of atomic spin-direction eigenfunctions exiting the magnet, not a superposition.

Decades ago, Wigner described a Stern-Gerlach thought experiment in which additional, downstream magnets would bend the two separated atomic beams back together [39]. Then, assuming, as usual, a spin superposition exiting the S.-G. magnet, he predicted an interference pattern resulting from the two overlapping wavepackets. In a very detailed analysis, however, Englert, Schwinger, and Scully [40] showed that it is not possible to so carefully construct a magnetic field that it would reunite such previously separated wavepackets. But, Qureshi and Rahman have recently suggested using a microfabricated, two-slit screen, instead of the additional magnets, to bring the two beams together again [41]. Each slit would be aligned with one of the two, separated streams of atoms, downstream of the S.-G. magnet, permitting just one spin-direction type to pass each slit. The slits are so narrow they would diffract, and diffuse, then overlap, each supposed atomic wavepacket. One could employ such an

\footnotetext{
${ }^{4}$ Because the total energy of the neutron is unchanged traversing a static, uniform magnetic field (not an S-G magnet) continuous Schrödinger evolution does describe that wavefunction.
} 
arrangement to test whether it is, indeed, a spin superposition, rather than a mixture, that exits the S.-G. magnet. If a superposition, there ought to be observable interference. ${ }^{5}$

Another very simple experiment can demonstrate that spin one-half atoms entering the S.-G. magnetic field immediately exchange energy with the field, and assume new energy eigenvalues. In the field there will be Zeeman splitting of that atom's energy levels. Consider, for example, sodium atoms, which, like silver, have a spin of one-half from a single valence electron, and can be used for an S.-G. observation. The ground-state absorption spectrum of sodium, absent any magnetic field, may be recorded first. The ionization energy is $5.18908 \mathrm{eV}$, such that light of a wavelength of 2412.59 Angstrom is sufficient to free the valence electron from the atom.

In the presence of a magnetic field of about 0.4 Tesla, the ionization energy will shift by about \pm $2.315 \times 10^{-5} \mathrm{eV}$, or about 5 parts in $10^{6}$. This splitting is, of course, visible in an absorption spectrum of sodium within the field. And a nearly monochromatic light source at 2412.59 \pm 0.01 Angstrom, focused on sodium atoms in the magnetic field, will display no absorption edge at this previous ionization energy, indicating an immediate energy exchange when the atom enters the magnetic field.

The ionization energy level of the sodium valence electron shifts by $\pm \mu_{z} B_{z}$ in the Stern-Gerlach magnet, indicating a spin parallel or anti-parallel to $\mathrm{B}_{\mathrm{z}}($ near $\mathrm{x}=0)$. The spin portion of the atom's wavefunction, $\frac{1}{\sqrt{2}}\left(\left|\uparrow_{z}\right\rangle+\left|\downarrow_{z}\right\rangle\right)$, before encountering the field, becomes either $\left|\uparrow_{z}\right\rangle$, or $\left|\downarrow_{z}\right\rangle$, in the field.

Notice that the nearly monochromatic light source does not measure either one of the two, new, energy levels of the atom. So, it is not a measurement of the new energy level, but, instead, immediate energy exchange with the $\mathbf{B}$ field, as in a Stern-Gerlach experiment, that reduces the spin superposition to a mixture.

\section{Comments}

When the explanation of some physical phenomenon has been accepted by researchers for many decades, it can be difficult to penetrate the conditioned comprehension so engendered. The influential philosopher of science, Thomas Kuhn, argued that, for some scientists, an actual gestalt switch may be necessary to accept new ideas that contradict long-inculcated, and abiding, though unverified, scientific theories [42]. Explanation of the Stern-Gerlach experiment may be such a case. It seems that for decades all our textbooks, and research articles, have persisted in assuming an S.-G. spin superposition, absent empirical evidence to exclude the alternative possibility of a mixture. Authentic scientific explanation is consistent within its realm of applicability. The ubiquitous, orthodox description of the Stern-Gerlach experiment, which posits continuation of a spin (and energy) superposition, cannot be valid for particles whose total energy is not conserved.

Through many decades of refinement, theories explaining the S.-G. experiment have become more rigorous, incorporating a realistic model of the magnetic field, and moving from a classical, to a fully quantum-mechanical description. But, all the extant explanations of the S.-G. phenomenon continue to presume continuous Schrödinger evolution during energy exchange with the observed particle, and, as such, are inadequate. As Feynman, Wigner, and others have taught us, energy exchange must reduce the

\footnotetext{
${ }^{4}$ Oddly, Qureshi and Rahman suppose that correlation of spin up with one of their two continuing wavepackets, beyond the S.-G magnet, and spin down with the other ("entanglement"), renders each wavepacket distinguishable. So, they predict the location of the spinning atom, as Feynman taught, by adding probabilities (absolute square of each wavepacket), not probability amplitudes. Thus, they suppose no interference downstream of the overlap-inducing, two-slit screen. Even with this peculiar supposition, however, one may still test whether a superposition, or mixture, exits the S.-G. magnet. With a second S.-G. magnet, rotated ninety degrees, beyond the two-slit screen, Qureshi and Rahman then predict an interference pattern at the detector screen. But, there will be no such interference, since each atomic wavefunction leaves the first S.-G. magnet as a single spatial wavepacket, along one path, not a superposition.
} 
observed wavefunction to a single measured eigenfunction, disrupting continuous Schrödinger time development.

We've seen that the actual, quantum-mechanical description of the S.-G. experiment is without "entanglement", or physical precession of the atom's magnetic moment, and is thus, remarkably straightforward. The computation of wavefunction development for the single, spin-direction wavepacket can be performed iteratively, and, as it must, does correctly predict the observed outcome of the S.-G. experiment.

Several experiments have been presented here which substantiate the argument that energy exchange between the atom and the S.-G. magnet is necessarily a quantum measurement. Energy transfer there implies information exchange, a discontinuity in Schrödinger evolution, and reduction of the wavefunction to one energy, and spin, eigenfunction. Moreover, Rolf Landauer, a successor to Brillion at the I.B.M. Watson laboratory, continued to insist that all information is actually physical [43]. That would seem to imply, one may suggest, that all measurements, all information exchanges between two physical objects, also require an energy exchange between those two.

\section{Appendix}

One might suppose that existence of a device called a Stern-Gerlach "interferometer" demonstrates that two superposed wavepackets must persist beyond the magnetic field in the S-G apparatus. That, in spite of energy exchange in the magnet's field, it is not a statistical mixture of silver atoms, but rather, a superposition of two spin-direction wavepackets, that is incident on the S-G detector. We shall see here that such is not the case.

A basic Stern-Gerlach "interferometer" is depicted in Fig. A.1. It is a device refined by Ramsey [32] from a simpler design used extensively by Rabi and his colleagues to measure magnetic moments [44]. Today, the apparatus, in modified form, is employed for a variety of experiments [33-36, 45]. Typically, atoms from the source will have a magnetic moment, $\mu_{\mathrm{A}}$, which permits two possible energy states, ground and excited, when they experience the uniform magnetic field, $\mathbf{B}_{\mathbf{U}}$. An atom's magnetic moment does not physically precess about $\mathbf{B}_{\mathbf{U}}$, but instead is quanitized parallel or anti-parallel to the field. However, there still exists an oscillatory component to the atom's wavefunction, namely, $\psi(\mathbf{r}, t)=\bar{\psi}(\mathbf{r}, t) e^{i \omega_{L} t}$, with $\omega_{\mathrm{L}}$ the Larmour frequency, $\omega_{L}=\frac{\mu_{A} B_{U}}{\hbar}$. That wavefunction component predicts the probability of spin flip in the magnetic field.

Bordering the extended $\mathbf{B}_{\mathbf{U}}$ field are two Stern-Gerlach magnets, $\mathrm{SG}_{1}$ and $\mathrm{SG}_{2}$. $\mathrm{SG}_{1}$ selects ground-state atoms and $\mathrm{SG}_{2}$ permits only excited atoms to reach the detector. A magnetic field, perpendicular to $\mathbf{B}_{\mathbf{U}}$, and rotating about it, is produced by the generator, RFG, in the two confined, narrow regions, $\mathrm{RF}_{1}$, and $\mathrm{RF}_{2}$, through which an atom must pass. The strength of $\mathrm{RFG}$, with a frequency, $\omega_{\mathrm{D}}$, near $\omega_{\mathrm{L}}$, may be adjusted so that a ground-state atom transiting the first interaction region will be equally likely to have jumped, or not, into its excited state. There is then a fifty percent probability it has absorbed a magnetic field quantum from the first rotating field, $\mathrm{RF}_{1}$.

At $\mathrm{RF}_{1}$ the oscillatory wavefunction component will be in phase with the rotating magnetic field, RFG. If RFG is exactly at the Larmour frequency, $\omega_{\mathrm{D}}=\omega_{\mathrm{L}}$, they will also be in phase at $\mathrm{RF}_{2}$, no matter the atom's translational velocity through the device. If RFG is not exactly at the Larmour frequency, however, the second rotating magnetic field will be out of phase with the atomic wavefunction oscillations there. And the probability that the atom will exchange a field quantum at $\mathrm{RF}_{2}$, and jump to its other energy state, is a sinusoidal function of the phase angle, $\phi_{\square}$, between the two. That phase angle is given by the formula,

$$
\phi_{R}=\operatorname{ToF}\left(\omega_{D}-\omega_{L}\right)
$$

with ToF the time of flight needed for an atom to travel between $\mathrm{RF}_{1}$ and $\mathrm{RF}_{2}$. 
If all atoms were traveling at exactly the same speed through the apparatus, then a plot of the rate of (excited) atoms at the detector versus the phase, $\phi_{\mathrm{R}}$, would look like the simple sinusoidal curve shown in part (a) of Fig. A.2 [46]. We note, with care, that this sinusoidal curve is decidedly not due to two superposed wavepackets, representing a single atom, resulting in an interference pattern. Not analogous, that is, to the interference pattern caused, say, by individual photon wavepackets passing through two separated, narrow slits. Instead, Fig. A.2, (a) depicts the periodic fluctuations of the transition probability to the atom's excited state, versus variation in $\phi_{\mathrm{R}}$.

Because atoms are supplied to this device with some limited range of velocities, there will be a range of flight times, and a range of $\phi_{\square}$ values, for a given frequency, $\omega_{\mathrm{D}}$. That spread of velocities results in the detection pattern shown in part (b) of Fig. A.2 [47]. The sharpness of this measured resonance permits a more precise determination of observed magnetic moments, for example.

There is about a fifty percent chance that the quantum of energy, $\hbar \omega_{L}$, will be absorbed by a ground-state atom at $\mathrm{RF}_{1}$, so promoting it to the excited energy state. Thus, it is often assumed that an individual atom's wavefunction is split in two there, with one spatial wavepacket, representing the ground-state atom, traveling to $\mathrm{RF}_{2}$, while simultaneously, a second wavepacket, representing the excited atom, moves along the identical path. But the sinusoidal curves of Fig. A.2, which have a superficial resemblance to interference fringes, are not due to a varying overlap of wavepackets from one atomic wavefunction. Instead, they show a sinusoidal dependence of the detection rate on the phase angle, $\phi_{\mathrm{R}}$, between the driving field and the wavefunction's Larmour frequency oscillations.

The extraordinary atomic fountain built by Kasevitch and Chu, [33] and employed by Kasevich and his colleagues, [36] utilizes the same principle as Ramsey's apparatus. Instead of a macroscopic magnetic field, like $\mathrm{B}_{\mathrm{U}}$, the magnetic field of an atom's own nucleus splits the atomic energy level (hyperfine splitting). Sodium atoms are used by Kasevich and Chu, and a $\pi / 2, \pi, \pi / 2$ sequence of polarized laser pulses induces the transition $\mathrm{F}=1, \mathrm{~m}_{\mathrm{F}}=0$ to $\mathrm{F}=2, \mathrm{~m}_{\mathrm{F}}=0$ in some of the atoms. Those atoms which are excited are then ionized and detected with a microchannel plate.

A plot of the rate of excited, detected atoms, versus frequency of the exciting laser pulse, results in a seemingly familiar sinusoidal curve [48]. One might assume the laser pulses produce a quantum energy superposition of the atom's excited and ground states. But there is, in fact, no phase difference along the path length of excited atoms, compared to that along the path of those remaining in the ground state. Instead, as in Ramsey's device, it is a variation of the phase of the electromagnetic pulse, versus phase of the atom's oscillating wavefunction component, which generates this sinusoidal transition probability. 

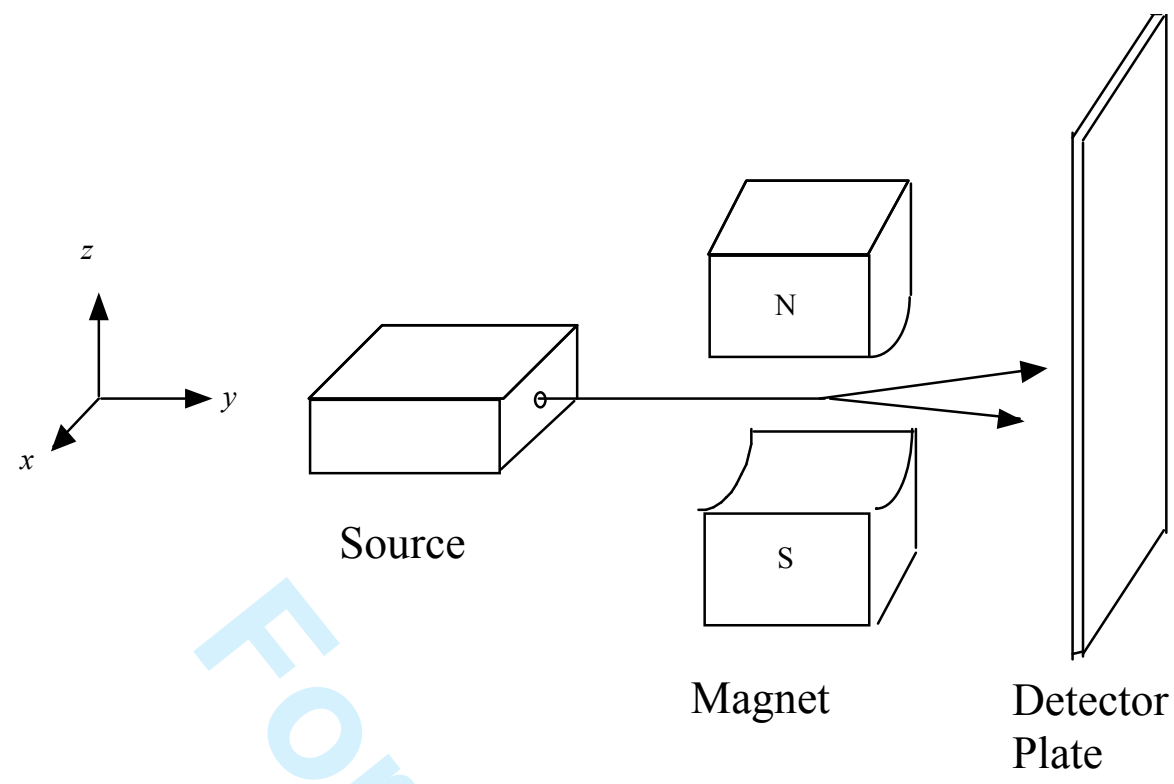

Magnet

Fig. 1. The diagram depicts, schematically, the Stern-Gerlach experiment, used to demonstrate that the magnetic moment of a silver atom is quanitized either parallel or anti-parallel to the magnetic field, $\mathbf{B}$.

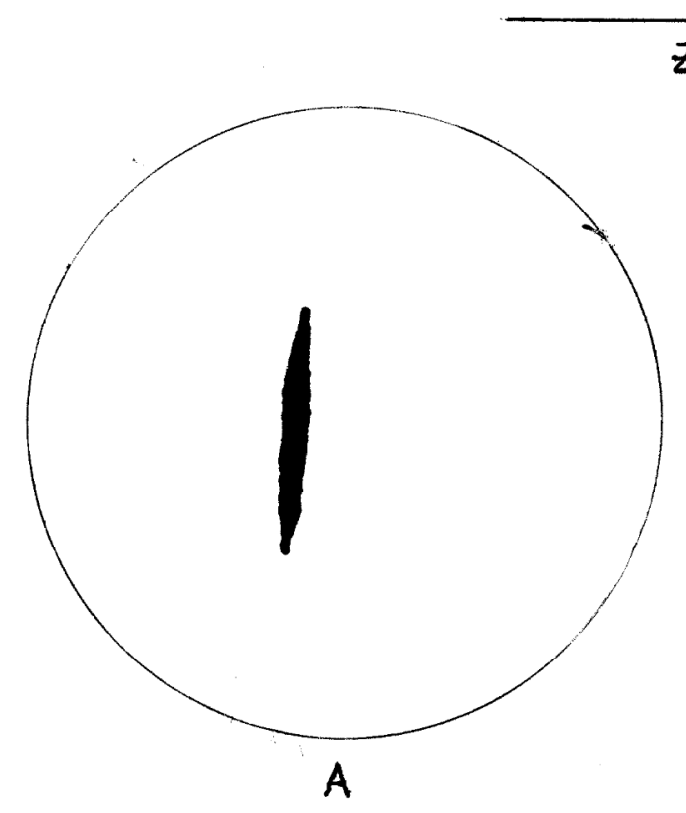

$z$

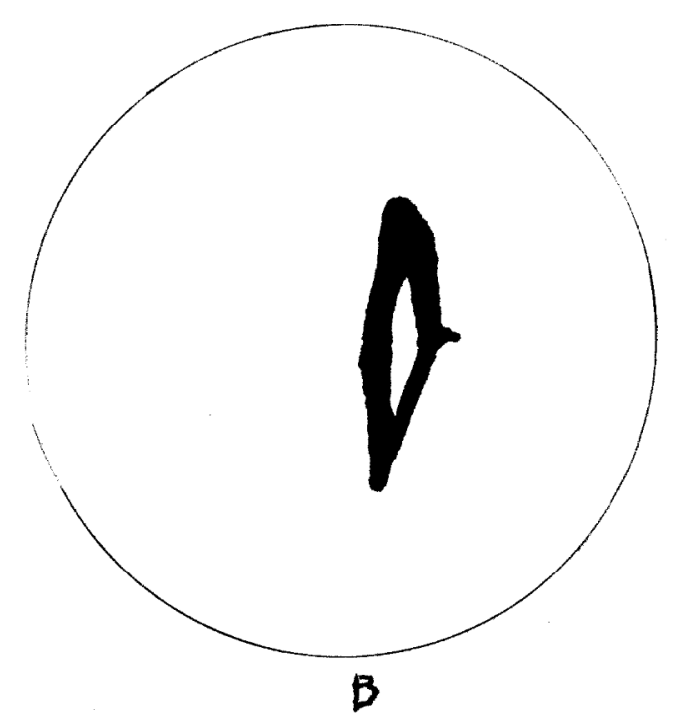


Fig. 2. Shown is a facsimile of the silver atoms detected by Stern and Gerlach downstream from the magnet. A. The silver atoms detected with the magnet turned off. B. The detected silver atoms when the magnetic field is on.

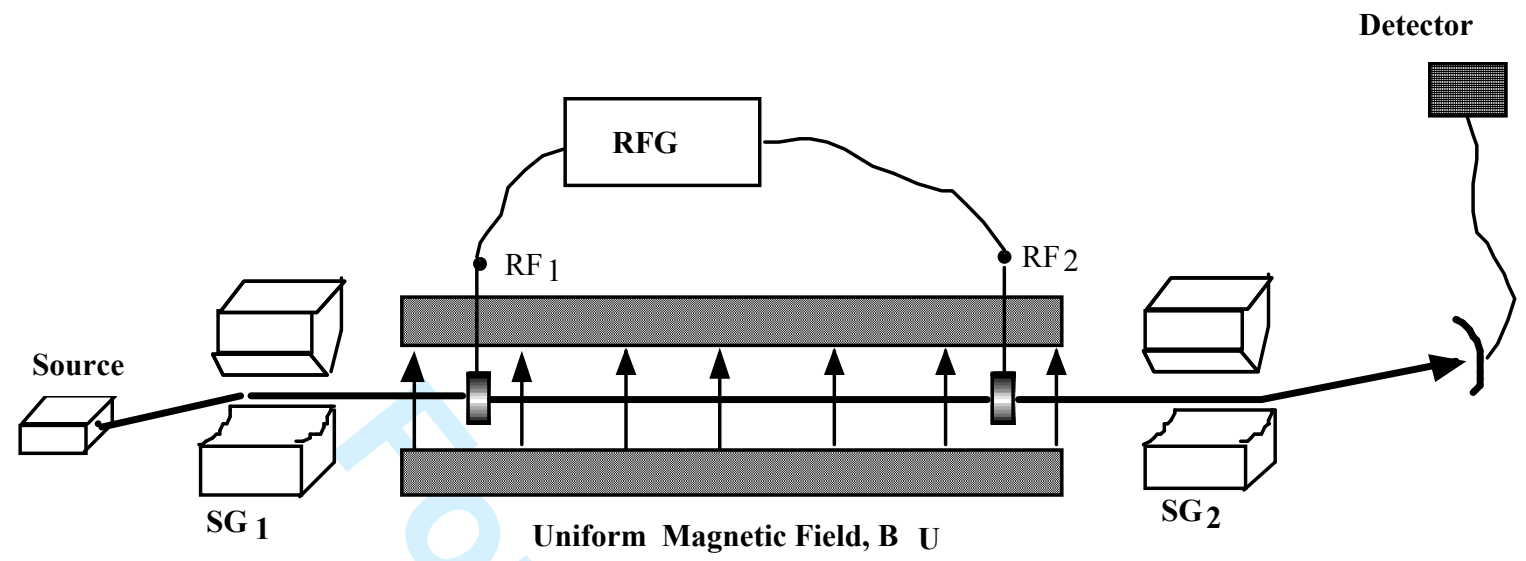

Fig. A.1. A basic Stern-Gerlach "interferometer" is shown schematically. Ground-state atoms from the source are selected by the magnet $\mathbf{S G}_{1}$. Those atoms, passing through the uniform field, $\mathbf{B}_{\mathbf{U}}$ experience a rotating magnetic field at $\mathrm{RF}_{1}$ and $\mathrm{RF}_{2}$, which may cause a transition to the excited energy level. Only excited atoms which pass through the $\mathrm{SG}_{2}$ magnet can reach the detector.

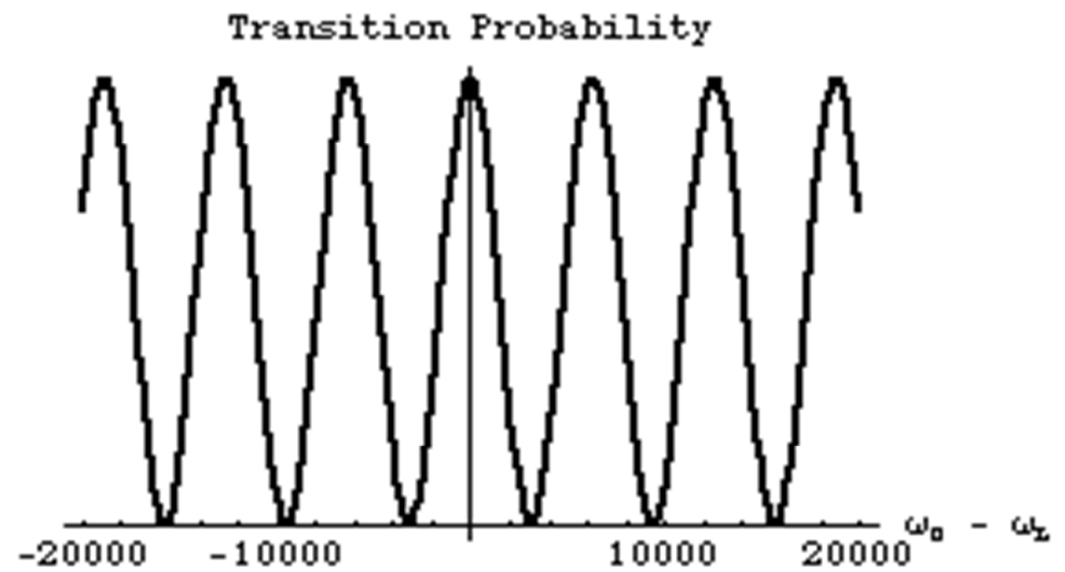

$(\alpha)$ 


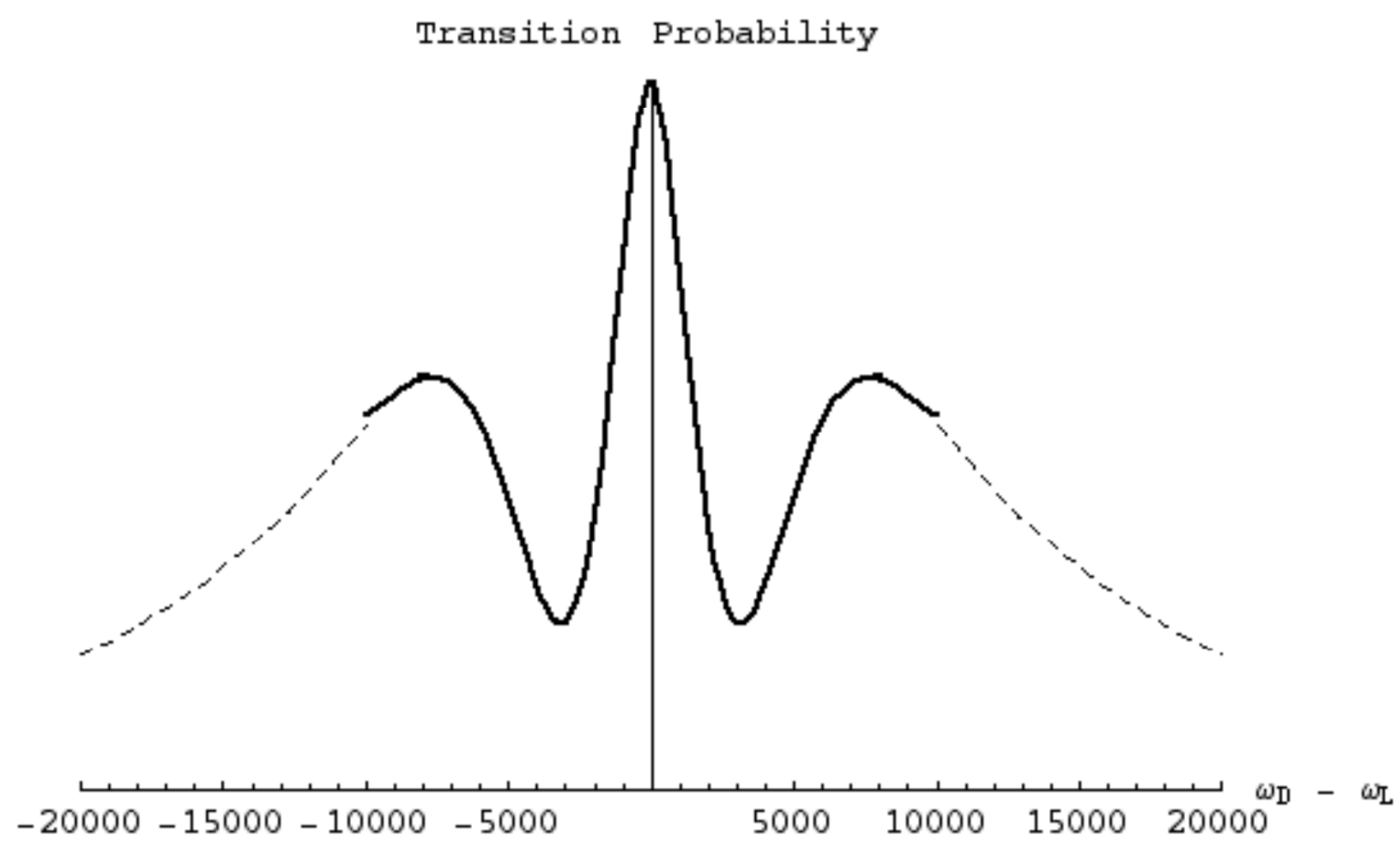

(b)

Fig. A.2. The probability for transition to the atom's excited state is shown as a function of the angular frequency, $\omega_{\mathrm{D}}$, of the rotating magnetic field generated in the confined regions, $\mathrm{RF}_{1}$, and $\mathrm{RF}_{2}$. Part (a) displays that probability if all atoms had a single velocity through the device. Part (b) depicts the result for a stream of atoms with a more realistic spread of incident velocities. The solid line is the transition probability near resonance, $\left|\omega_{D}-\omega_{L}\right| \leq 1 \times 10^{4} \mathrm{rad}$. / sec. The dashed line is an extrapolation for the offresonance transition probability.

\section{References}

1. O. Stern. Z. Phys. 7, 249 (1921). 
2. W. Gerlach and O. Stern. Z. Phys. 9, 349 (1922).

3. S.H. Patil. Eur. J. Phys. 19, 25 (1998).

4. G.B. Roston, M. Casas, A. Plastino, and A.R. Plastino. Eur. J. Phys. 26, 657 (2005).

5. $\quad$ B.C. Hsu, M. Berrondo, and J.-F.S. Van Huele. Phys. Rev. A 83, 012109 (2011).

6. H. Wennerstrom and P.-O.Westlund. Phys. Essays 26, 174 (2013).

7. R. Feynman, R. Leighton, and M. Sands. The Feynman Lectures on Physics, Vol. III. AddisonWesley. 1965. Chp. 3-3.

8. $\quad$ Ref. [7], Chp. 3.

9. D. Bohm. Quantum Theory. Prentice-Hall. 1951. p. 596.

10. H. Rauch. Phys. Scripta T76, 24 (1998).

11. K. Hornberger. Revs. Mod. Phys. 84, 157 (2012).

12. See, J. Von Neumann. Mathematical Foundations of Quantum Mechanics. Chp V. Princeton University Press. 1955.

13. E.P. Wigner in "Introduction to Quantum Mechanics," reprinted in J. A. Wheeler and W. Zurek, Quantum Theory and Measurement. Princeton University Press. 1983. II.2, p. 267.

14. W.H. Zurek. Physics Today 36 (October, 1991).

15. D. Bohm. Quantum Theory. Prentice-Hall. 1951. Chp. 22.

16. R. Leighton. Principles of Modern Physics. McGraw-Hill. 1959. Chp. 2.

17. M. Devereux Found. Phys. Lett. 16, 41 (2002).

18. M. Born. Z. Phys. 37, 863 (1926).

19. Ref. [15], p. 593-8.

20. M.O. Scully, R. Shea, and J.D. McCullen. Phys. Reports 43C, 485 (1978).

21. E. Kennard. Z. Phys. 44, 326 (1927).

22. M. Devereux, "Testing Quantum Erasure and Reversible Quantum Measurement with Holladay's Simple Experiment", In $6^{\text {th }}$ International Symposium on Quantum Theory and Symmetries.

Edited by S. Das and A. Shapere. 2013 J. Phys.: Conf. Ser. 462011001.

23. M.O. Scully, W.E. Lamb, and A.Barut Found. Phys. 17, 575 (1987).

24. P. Alstrom, P. Hjorth, and R. Mattuck. Am. J. Phys. 50, 697 (1982).

25. D. E. Platt. Am. J. Phys. 60, 306 (1992).

26. D. Home, A.K. Pan, M.M. Ali, and A.S. Majumdar. J. Phys. A 40, 13975 (2007).

27. G. Potel, F. Barranco, S. Cruz-Barrios, and J. Gomez-Camacho. Phys. Rev. A 71, 052106 (2005).

28. S. Cruz-Barrios and J. Gomez-Camacho. Nucl. Phys. A 636, 70 (1998).

29. Ref. [5], p. 9.

30. B. Alefeld, G. Badurek, and H. Rauch, Phys. Lett. 83A, 32 (1981).

31. Personal communications.

32. N. F. Ramsey. Phys. Rev. 78, 695 (1950).

33. M. Kasevich and S. Chu. Phys. Rev. Lett. 67, 181 (1991).

34. Ch.J. Borde, N. Courtier, F. du Bruck, A.N. Goncharov, and M. Gorlivki. Phys. Lett. A 188, 187 (1994).

35. K. Eckert, P. Hyllus, D. Bruss, U.V. Poulsen, M. Lewenstein, C. Jentsch, T. Muller, E.M. Rasel, and W. Ertmer. Phys. Rev. A 73, 013814 (2006).

36. S. Dickerson, J. Hogan, A. Sugarbaker, D. Johnson, and M. Kasevich. Phys. Rev. Lett. 111, 083001 (2013).

37. Ref. [7], Chp. 3-3.

38. J.M. Jauch, "Foundations of Quantum Mechanics". In Foundations of Quantum Mechanic, Edited by B. d'Espagnet. p. 39. Academic Press. 1971.

39. E. P. Wigner. Am. J. Phys. 31, 6 (1963).

40. B.-G. Englert, J. Schwinger, and M.O. Scully. Found. Phys. 18, 1045 (1988).

41. T. Qureshi and Z. Rahman. Prog. Theo. Phys. 127, 71 (2011).

42. T. Kuhn. The Structure of Scientific Revolutions. University of Chicago Press. 1970.

43. R. Landauer. Phys. Today, 23 (May, 1991) 
44. I.I. Rabi. Phys. Rev. 51, 652 (1937).

45. K. Rubin, M. Eminyan, F. Perales, R. Mathevet, K. Brodsky, B. Viaris de Lesegno, J. Reinhardt, M. Boustimi, J. Baudon, J.-C. Karam, and J. Robert. Laser Phys. Lett. 1, 184 (2004).

46. See Ramsey's Figure 5, N. F. Ramsey. Phys. Today, 25 (July, 1980), p. 27.

47. See Ramsey's Figure 2, Ref. [32], p. 697.

48. Ref. [33], Figure 3, p. 183. 